Copyright by the Optical Society of America. Hong Di, Kangfeng Zheng, Xin Zhang, Edmund Y. Lam, Taegeun Kim, You Seok Kim, Ting-Chung Poon, and Changhe Zhou, "Multiple-image encryption by compressive holography," Appl. Opt. 51, 1000-1009 (2012); doi: $10.1364 / \mathrm{AO} .51 .001000$

\title{
Multiple-image encryption by compressive holography
}

\author{
Hong Di, ${ }^{1}$ Kangfeng Zheng, ${ }^{1}$ Xin Zhang, ${ }^{2, *}$ Edmund Y. Lam, ${ }^{3}$ Taegeun Kim, ${ }^{4}$ \\ You Seok Kim, ${ }^{4}$ Ting-Chung Poon, ${ }^{5,6}$ and Changhe Zhou ${ }^{6}$ \\ ${ }^{1}$ Information Security Center, Beijing University of Posts and Telecommunications, Beijing 100876, China \\ ${ }^{2}$ National Laboratory of Pattern Recognition, Institute of Automation, Chinese Academy of Sciences, \\ Beijing 100190, China \\ ${ }^{3}$ Imaging Systems Laboratory, Department of Electrical and Electronic Engineering, \\ University of Hong Kong, Pokfulam Road, Hong Kong, China \\ ${ }^{4}$ Department of Optical Engineering, Sejong University, 98 Kunja-dong, Kwangjin-gu, Seoul 134-747, South Korea \\ ${ }^{5}$ Bradley Department of Electrical and Computer Engineering, Virginia Tech, Blacksburg, Virginia 24061, USA \\ ${ }^{6}$ Shanghai Institute of Optics and Fine Mechanics, Chinese Academy of Sciences, \\ P.O. Box 800-211, Shanghai 201800, China \\ ${ }^{*}$ Corresponding author: xzhang @ nlpr.ia.ac.cn
}

Received 19 September 2011; revised 10 January 2012; accepted 10 January 2012; posted 10 January 2012 (Doc. ID 154976); published 1 March 2012

\begin{abstract}
We present multiple-image encryption (MIE) based on compressive holography. In the encryption, a holographic technique is employed to record multiple images simultaneously to form a hologram. The twodimensional Fourier data of the hologram are then compressed by nonuniform sampling, which gives rise to compressive encryption. Decryption of individual images is cast into a minimization problem. The minimization retains the sparsity of recovered images in the wavelet basis. Meanwhile, total variation regularization is used to preserve edges in the reconstruction. Experiments have been conducted using holograms acquired by optical scanning holography as an example. Computer simulations of multiple images are subsequently demonstrated to illustrate the feasibility of the MIE scheme. (C) 2012 Optical Society of America
\end{abstract}

OCIS codes: $\quad 060.4785,090.1995,090.1760,100.3020,100.3190$.

\section{Introduction}

Optics-based methods take advantage of processing two-dimensional (2D) data in parallel and have been studied in the context of optics and information security. Among the many proposed techniques, one of the prominent optical techniques is double random phase encryption [ $\underline{1}-\underline{3}$ ]. The system adopts two random phase masks to encrypt the input image into stationary white noise. However, in the last decade, optical encryption systems have been evolving into multiple-image encryption (MIE), which attracts

$1559-128 \mathrm{X} / 12 / 071000-10 \$ 15.00 / 0$

(C) 2012 Optical Society of America much attention nowadays owing to economic memory occupation and efficient transmission via a network. Compared with single-image encryption, MIE encodes several images into a single encrypted file [4-7]. Wavelength multiplexing has been used in the context of MIE [5]. The ultimate encrypted image was synthesized by superimposing individual encrypted image together. This encryption strategy was time-consuming and sensitive to the cross-talk effect. MIE based on a frequency shift was also proposed to encode images in either the Fourier or fractional Fourier domain. The technique is good at encoding multiple images, but high-frequency contents of the images have to be discarded due to downsize cropping of the spectrum in order to utilize the 
algorithm effectively $[4,8]$. MIE based on the fractional Fourier transform (FT) using different fractional orders [6] has been used, but the method is time-consuming as it produces initial phase terms by iterations.

Holography was also involved in image encryption because of the inherent capability of recording images into a hologram. A hologram can preserve the image content by its complex Fresnel zone patterns, and the encryption procedure can be performed by real optical systems [7,9-12]. Holographic techniques have been used to encrypt a single image or a three-dimensional (3D) object. However, hologram data are not only complex but also occupy much more memory than does the original image. A plethora of publications in the past decade have dealt with the encryption of holograms [13-16]. Investigations of encrypted holograms using compressive sensing, however, have not been explored to the best of our knowledge. In this paper, we believe we present the first work dealing with MIE by compressive holography. In order to achieve an efficient MIE, we utilize optical scanning holography $(\mathrm{OSH})$ to encrypt multiple images and introduce compressive sensing (CS) to perform compressive encryption. Decryption is treated as a minimization problem.

In the encryption process by $\mathrm{OSH}$, a hologram is recorded to encode the information of multiple images. A nonuniform sampling is then applied on the Fourier result of the hologram to produce the compressed hologram. Subsequently, decryption is expressed as a minimization problem [17-20]. In the CS framework, the solution to the problem preserves the sparsity of the images as well as fidelity by an $l_{2}$-norm constraint. As the encryption/decryption process handles natural images, total-variation regularization is added to preserve edges. Finally, the decryption process ensures images are recovered with edges preserved.

In what follows, we will first present the methodology in Section 2. It starts with the model of MIE by $\mathrm{OSH}$, discusses the compression of the encrypted hologram by CS, and finally moves to the minimiza- tion problem of the decryption of images. Section 3 describes two experiments: the first one deals with optical experiments on the encryption of hologram data of two images, and the other one works on the simulation of a multiple-image hologram. Concluding remarks are summarized in Section 4 .

\section{Methodology}

\section{A. Optical Scanning Holography}

OSH records the holographic information of a 3D object on a $2 \mathrm{D}$ hologram by $2 \mathrm{D}$ optical heterodyne scanning. The technique was pioneered by Poon [21]. A standard experimental setup is shown in Fig. 1 . A laser at temporal frequency $\omega$ goes into the system. It is split by a beam splitter $\left(\mathrm{BS}_{1}\right)$ into two optical beams. One beam passes through the mirror $\left(\mathrm{M}_{1}\right)$, the pupil $\left[l_{1}(x, y)\right]$, and the lens $\left(\mathrm{L}_{1}\right)$. The other beam is frequency-shifted by an acousto-optical frequency shifter (AOFS) to frequency $\omega+\alpha$. The frequencyshifted optical beam is then propagating through the mirror $\left(\mathrm{M}_{2}\right)$, the pupil $\left[l_{2}(x, y)\right]$, and the lens $\left(\mathrm{L}_{2}\right)$ successively as shown in Fig. 1 . The two optical beams are then combined by a beam splitter $\left(\mathrm{BS}_{2}\right)$ and controlled by the $x-y$ scanner to scan an object at $z$ away from the scanning system. The scattered and diffracted light from the object is collected by lens $\mathrm{L}_{3}$ and converged onto a photodetector (PD), which records the optical information of the object and converts it into an electronic signal. The postprocessing unit processes the electronic signal and generates scanned information of the object in a computer [22]. If the pupil functions $l_{1}(x, y)$ and $l_{2}(x, y)$ are of the form of an open mask and a pin hole, respectively, what is being processed and stored in a computer are two digital holograms [23].

\section{B. Compressive Sensing}

CS brings a novel technique to image compression. It is based on the inherent sparsity of images and can recover compressed images with desirable quality from much fewer compressed data. In our case, the technique will be applied to the hologram captured

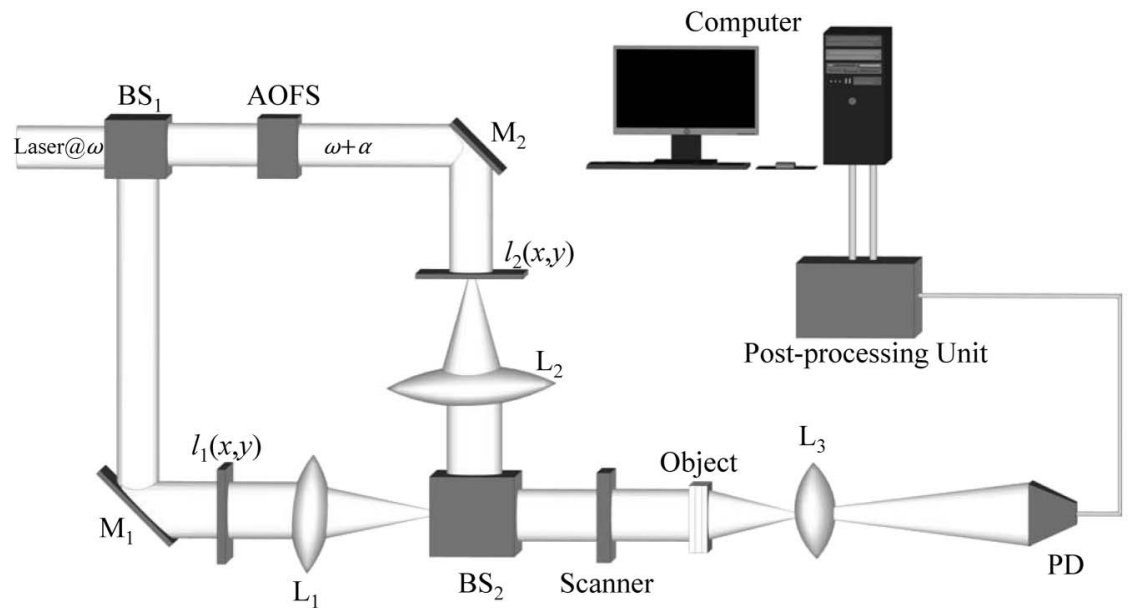

Fig. 1. Standard experimental setup of OSH. 
by the OSH system. Before that, there is a need to explain CS concisely.

Suppose there is an image, $S$, to be compressed. It is of size $N \times N$. The image is organized into a vector in the lexicographical order, $\mathbf{s} \in \mathbb{R}^{N^{2}}$. To implement $\mathrm{CS}$ on the vector is to design a sensing operator, which makes a projection of the vector from $\mathbb{R}^{N^{2}}$ to $\mathbb{R}^{M}$. Given the sensing operator $\Phi \in \mathbb{R}^{M \times N^{2}}$ and $M \ll N^{2}$, ss will achieve the compression of the vector $\mathbf{s}$ from $\mathbb{R}^{N^{2}}$ to $\mathbb{R}^{M}$. That is,

$$
\mathbf{g}=\Phi \mathbf{s}
$$

Equation (1) conducts the sensing projection to achieve a compression of the data. The next step is to recover the image from the compressed data, $\mathbf{g}$. In compressive sensing, there are two sufficient conditions to guarantee that there is an optimal reconstruction of the original image. The first one is the transform sparsity, which requires that the sparsity of the image is exposed under a transform, such as a bounded variation and wavelet transform. For example, the wavelet transform realizes the sparse image representation and can be denoted by $\Psi$, that is, $\mathbf{x}=$ $\Psi^{H} \mathbf{S}$ produces the sparse vector $\mathbf{x}$ from a vector $\mathbf{s}$. Here the superscript $H$ denotes the Hermitian transpose. The second sufficient condition is that the sensing operator and the sparse representation operator satisfy the restricted isometry property,

$$
(1-\epsilon)\|\mathbf{x}\|_{2}^{2} \leq\|\Phi \Psi \mathbf{x}\|_{2}^{2} \leq(1+\epsilon)\|\mathbf{x}\|_{2}^{2} .
$$

Here $\|\cdot\|_{2}$ stands for the $l_{2}$-norm of a vector, and $\epsilon$ is close to zero. Using the above condition, Zhang, Lam, and Poon pioneered CS in the context of holography [17]. There are a few pairs of transform operators that satisfy the condition. Among the many pairs, the Fourier-wavelet pair is suitably chosen for the MIE application. While the FT works as the sensing operator, the wavelet transform reveals the sparsity of the image. In the setting, reconstruction is obtained by minimizing

$$
f(\mathbf{x})=\lambda\|\mathbf{x}\|_{1}+\frac{1}{2}\|\mathbf{g}-\Phi \Psi \mathbf{x}\|_{2}^{2}
$$

where $\|\mathbf{x}\|_{1}=\sum_{i}\left|x_{i}\right|$ is the $l_{1}$-norm, which measures the number of nonzero entries of the vector $\mathbf{x}$, and $\lambda$ is the regularization parameter, which tunes the balance between the fidelity and regularization in the reconstruction.

\section{Compressive Hologram of Multiple Images}

The transfer function of the OSH system has been expressed as $[\underline{23}, \underline{24}]$

$$
H\left(k_{x}, k_{y}\right)=\exp \left[\frac{-j z}{2 k_{0}}\left(k_{x}^{2}+k_{y}^{2}\right)\right] .
$$

Here $z$ stands for the distance between the object and the holographic recording system, and $k_{0}$ is the wavenumber. Suppose an image is $s(x, y)$ and its FT is $S\left(k_{x}, k_{y}\right)$. The hologram measured by OSH can be represented by [23]

$$
c(x, y)=\mathcal{F}_{x y}^{-1}\left\{S\left(k_{x}, k_{y}\right) H\left(k_{x}, k_{y}\right)\right\} .
$$

Here $\mathcal{F}_{x y}^{-1}\{\cdot\}$ denotes the inverse FT operation. Suppose the 3D FT of an object is $\hat{Q}\left(k_{x}, k_{y}, k_{z}\right)$. The FT of the hologram, $C\left(k_{x}, k_{y}\right)$, is shown to be related to the 3D FT of the object as [25]

$$
\begin{aligned}
C\left(k_{x}, k_{y}\right) & =\hat{Q}\left[k_{x}, k_{y},-\left(k_{z}-k_{0}\right)\right] \\
& =\hat{Q}\left(k_{x}, k_{y}, k_{0}-\sqrt{k_{0}^{2}-k_{x}^{2}-k_{y}^{2}}\right) .
\end{aligned}
$$

In words, the samples of $C\left(k_{x}, k_{y}\right)$ comprise points equidistant from the point $\left(0,0, k_{0}\right)$ in the $3 \mathrm{D}$ spatial frequency domain, as shown in Fig. 2. Hence the data of the FT hologram represent a particular sampling of the 3D FT of the original object, and the FT result of a multiple-image hologram is equivalent to the partial Fourier data of these multiple images located at different places.

In order to perform CS of the hologram $c(x, y)$, sampling is applied on the FT result of the hologram. However, the sampling scheme should be designed particularly as the information distribution in the spatial frequency domain is nonuniform. If sampling is uniform, it fails to record sufficient information for subsequent reconstruction [26]. For well-designed sampling, more data should be measured around the origin, while less are picked out on the periphery. Thus the sampling pattern on the Fourier plane should appear as shown in Fig. 3, in which white points represent positions of sampled data. Note that the sampling pattern does not work on the 3D Fourier domain but on the 2D FT result of the hologram. A sampling ratio is defined as the number of sampling points divided by the number of overall resolvable points. Figure 3 shows a sampling pattern with the sampling ratio of $25 \%$. The sampling pattern is gradually less dense from the center to the periphery.

\section{Quantization of the Hologram}

The hologram can be stored as a signed IEEE 64-bit (8-byte) double-precision floating-point number. Compared with two 8-bit gray-scale bitmap images,

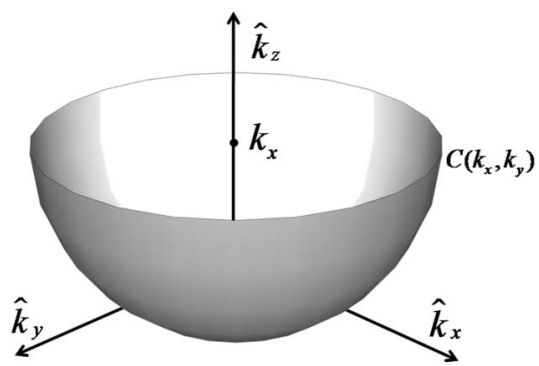

Fig. 2. Fourier data of a hologram, $C\left(k_{x}, k_{y}\right)$, exhibited in the 3D Fourier domain [25]. 


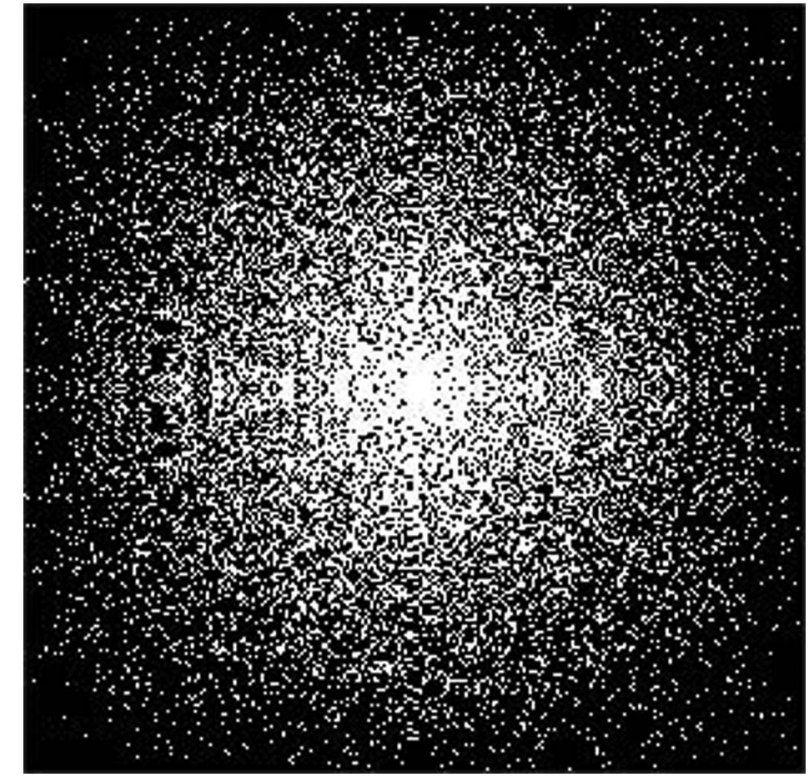

Fig. 3. Sampling pattern on the Fourier plane with sampling ratio of $25 \%$.

the size of hologram data are quadruply increased because of the double data type, $64 /(8 \times 2)=4$. In order to obtain a compressed hologram, not only do we need to employ sampling, but we should also consider quantization, which reduces the precision of the data. There are four choices: double, int32, int16, and int8. The last three types represent integer 32-bit, integer 16-bit, and integer 8-bit and occupy 4,2 , and 1 byte(s), respectively. So the quantization and compressive sensing ratio (QCSR) can be calculated as

$$
\mathrm{QCSR}=\frac{\text { Compressed-DataSize }}{\text { Original-ImageSize }}=\frac{2 r q}{l} .
$$

Here $l$ is the number of images in the encryption, $r$ is the sampling ratio, and $q$ is the bytes occupied by the data type used. Because a hologram is stored in real and imaginary parts, a factor of 2 is multiplied in Eq. (7). Both the quantization and sampling rate give rise to compressive hologram data. The peak signalto-noise ratio (PSNR) of the recovered image is measured to evaluate reconstruction with regard to the QCSR:

$$
\mathrm{PSNR}=10 \log _{10}\left(\frac{1}{\mathrm{MSE}}\right)
$$

with the MSE, denoting the mean square error, given by

$$
\operatorname{MSE}=\frac{1}{N \times N} \sum_{i=1}^{N} \sum_{j=1}^{N}\left(\hat{\mathbf{p}}_{i, j}-\mathbf{p}_{i, j}\right)^{2} .
$$

Here $\hat{\mathbf{p}}$ stands for the original image, and $\mathbf{p}$ is the recovered image. They are of the size $N \times N$.

\section{E. Discrete Wavelet Transform}

As the wavelet transform is used to sparsify an image in the work, here is a short introduction about the transform. A one-dimensional discrete wavelet transform (DWT) is adopted to illustrate the transform. Two-dimensional DWT of an image is described in a block diagram.

A DWT decomposes a signal into two parts, approximation and details, by the following pair:

$$
\begin{aligned}
X_{u, v} & =\sum_{n=-\infty}^{\infty} x(n) \phi_{u, v}(n) \quad \text { and } \\
D_{u, v} & =\sum_{n=-\infty}^{\infty} x(n) \psi_{u, v}(n) .
\end{aligned}
$$

Here $X_{u, v}$ and $D_{u, v}$ are referred to as the approximation coefficients and detail coefficients at scale and location indices $(u, v)$, respectively. Function $\phi_{u, v}(n)$ is called the scaling function and performs as a low-pass filter to obtain an approximation of the original signal, $x(n)$. It is defined as $\phi_{u, v}(n)=$ $2^{-u / 2} \phi\left(2^{-u} n-v\right)$ based on the father scaling function, $\phi(n)$. Function $\psi_{u, v}(n)$ is the wavelet function and works as a high-pass filter to obtain details of the signal, given by $\psi_{u, v}(n)=2^{-u / 2} \psi\left(2^{-u} n-v\right)$ based on the mother wavelet function, $\psi(n)$. A Daubechies wavelet is often used in image processing, but it is not possible to write down in closed form. Nevertheless, the father scale function and mother wavelet function of Daubechies D5 can be drawn. As shown in Fig. 4, the blue dashed curve is the wavelet function and the green curve is the scaling function. The index number, D5, refers to the number 5 of coefficients in the wavelet.

An example of the diagram of the decomposition of an image by the Daubechies D5 is shown in Fig. 5 . The original image of Lena is of the size of $256 \times 25 \overline{6}$. The rows of the image are convolved with $\phi(n)$ and $\psi(n)$, and the image is decomposed into horizontal approximation and horizontal detail at first. Then the horizontal approximation is decomposed further into the approximation and vertical detail by $\phi(n)$

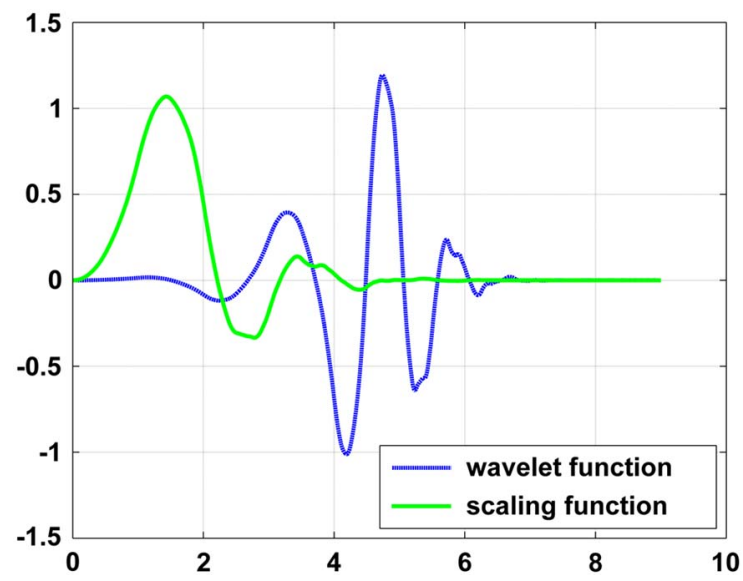

Fig. 4. (Color online) Daubechies D5 wavelet. 


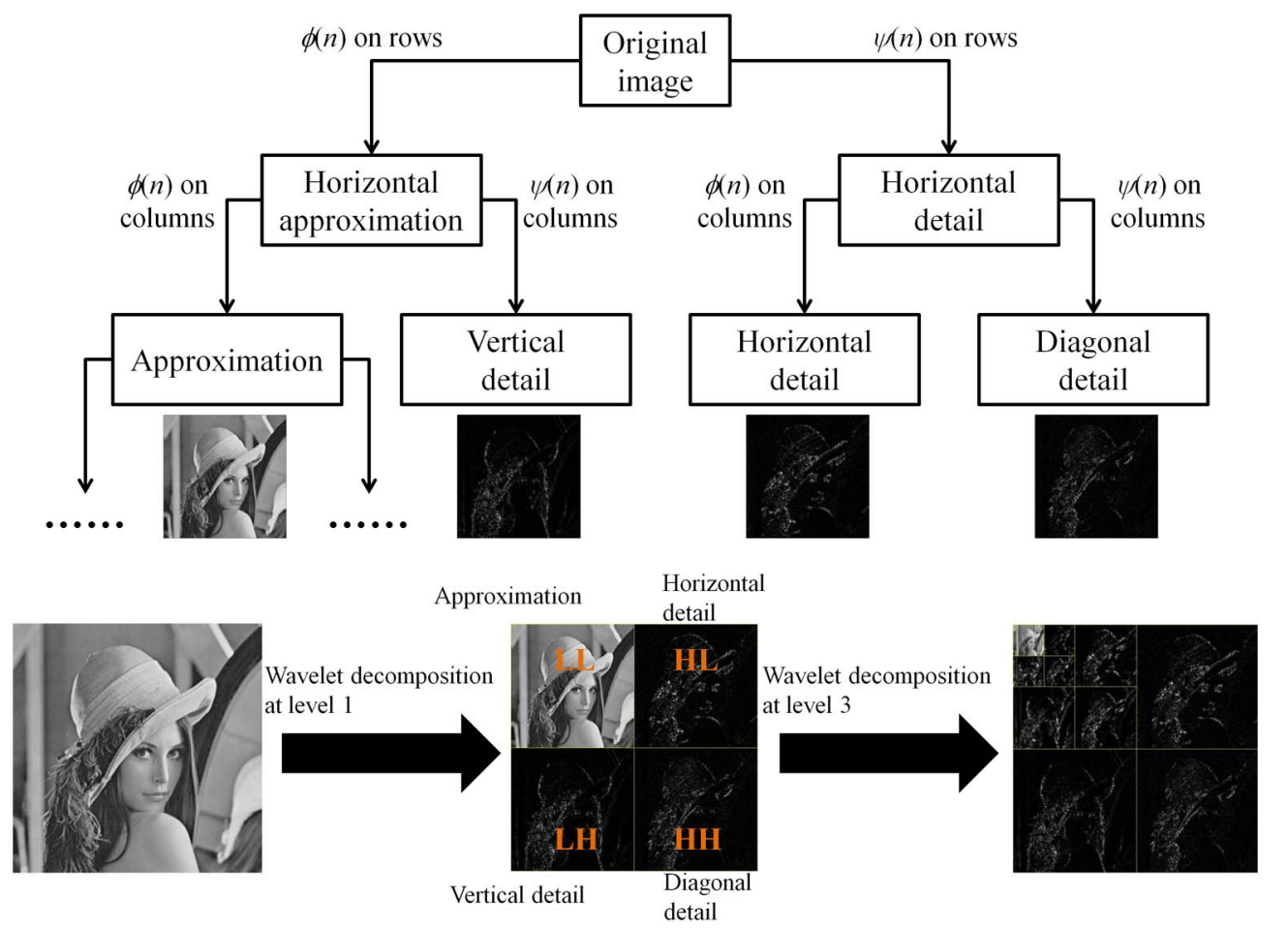

Fig. 5. (Color online) Diagram of the wavelet decomposition of Lena. The upper row shows the block diagram of the decomposition at level 1 , and the lower row shows the process and results at level 1 (middle figure) and level 3 (figure on the right side).

and $\psi(n)$ operated on columns, while the horizontal detail is decomposed into horizontal detail and diagonal detail also by $\phi(n)$ and $\psi(n)$ operated on columns. The four resulting subimages, that is, approximation, horizontal detail, vertical detail, and diagonal detail, compose the result of the decomposition at level 1 . The whole process is also shown in the lower row of Fig. 5, where the four subimages are arranged in the four quadrants in the decomposition and represented by $\mathrm{LL}, \mathrm{HL}, \mathrm{LH}$, and $\mathrm{HH}$, respectively. $\mathrm{L}$ and $\mathrm{H}$ stand for going through low-pass $\phi(n)$ and high-pass filter $\psi(n)$ for the corresponding subimages. For example, the left upper quadrant is denoted as LL because the original image is processed by $\phi(n)$ twice.

A decomposition at level 2 is operated on the approximation subimage, that is, on the quadrant LL. It separates the subimage into smaller subimages with approximation and three details similar to what is shown in level 1. A final result arising from the decomposition at level 3 is shown at the right lower corner of Fig. 5. Decomposition at level $k$ means the further decomposition of the approximation subimage at the level of $k-1$.

\section{F. Decryption Model}

Because the OSH system is linear and spaceinvariant, the model of encryption could then be constructed as

$$
\mathbf{c}=\mathbf{A s},
$$

where $\mathbf{c}$ and $\mathbf{s}$ are vectors denoting the compressed FT hologram and the original images in the lexico- graphical order, respectively. A is a matrix operator, which stands for sensing using OSH in this application. As we employ FT to compress the hologram, the model will be

$$
\tilde{\mathbf{c}}=\text { FAs }
$$

Here $\mathbf{F}$ denotes the FT operator.

Natural images possess significant sparsity under a wavelet basis. Let us take an example of the Lena image of the size $256 \times 256$. Under the Daubechies D5 wavelet decomposition at level 3 , the distribution of its coefficients is shown in Fig. 6(a). Here red lines indicate level 1, yellow lines show level 2, and green lines correspond to level 3. A 3D view of the decomposition results is shown in Fig. 6(b), where the $x$ and $y$ axes correspond to the vertical and horizontal directions in Fig. 6(a). Here we set the origin of Fig. 6(b) at the left upper point of Fig. 6(a). The $z$ axis indicates the value of decomposition coefficients. We observe that values of most of the coefficients, especially within the gray part of Fig. 6(a), are around zero, and these coefficients are significantly sparse. Figure 6(c) shows the coefficients of the Cameraman image under the same decomposition. The sparsity of coefficients demonstrates that the wavelet basis is an appropriate sparsity operator on natural images. Note that the Daubechies wavelet is not an exclusive choice for the application. Other kinds of wavelets are feasible as needed because the sparsity of natural images happens under most wavelets [27].

Referring to the CS theory, the pair of Fourier and wavelet basis is incoherent [28], which guarantees a 


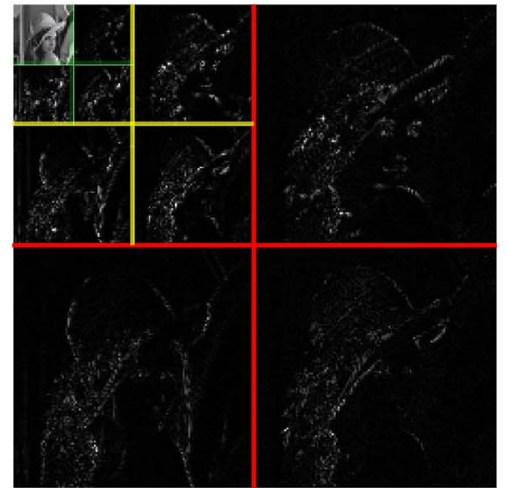

(a)

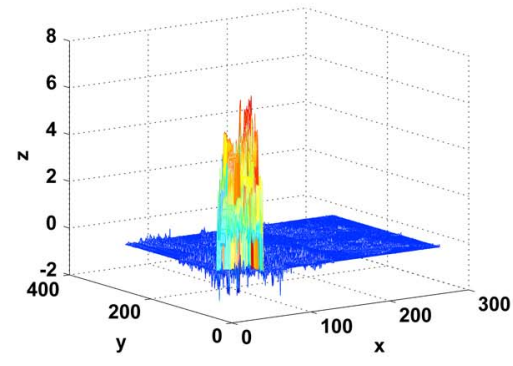

(b)

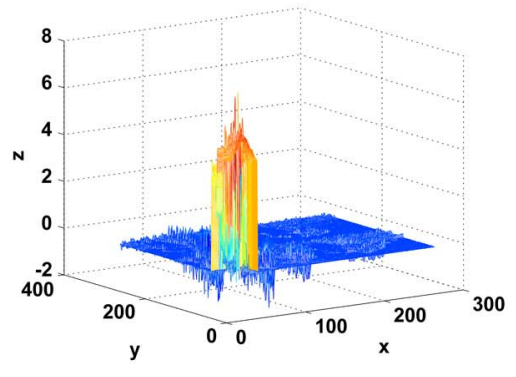

(c)

Fig. 6. (Color online) (a) Level 3 decomposition of Lena. (b), (c) Top view of the wavelet coefficient distribution in 3D view for Lena and Cameraman, respectively.

reconstruction from the compressed FT signal by $l_{1}$-norm minimization under the wavelet basis. Hence the decryption problem can be solved by $l_{1}$-norm minimization of

$$
f(\mathbf{x})=\alpha\|\Psi \mathbf{x}\|_{\mathbf{T V}}+\beta\|\mathbf{x}\|_{1}+\frac{1}{2}\|\tilde{\mathbf{c}}-\mathbf{F A} \Psi \mathbf{x}\|_{2}^{2} .
$$

Here $\|\cdot\|_{\mathrm{TV}}$ is the total variation (TV) regularization and

$$
\|q\|_{\mathrm{TV}}=\sum_{i, j} \sqrt{\left(q_{i+1, j}-q_{i, j}\right)^{2}+\left(q_{i, j+1}-q_{i, j}\right)^{2}} .
$$

Due to the nondifferentiability of the Euclidean norm at the origin, we will take an approximated TV as follows:

$$
\|q\|_{\mathrm{TV}}=\sum_{i, j} \sqrt{\left(q_{i+1, j}-q_{i, j}\right)^{2}+\left(q_{i, j+1}-q_{i, j}\right)^{2}+\xi^{2}} .
$$

Here $\xi$ is a small positive parameter. Apart from the consideration of sparsity and fidelity for a given reconstruction, TV regularization preserves edges of the recovered image while suppressing any spurious high-frequency components. After setting up the cost function, fixed-point iteration is employed to extract a solution for the problem.

\section{Experiments}

Two experiments were conducted to evaluate the performance of reconstruction under the compression of a hologram captured by OSH and of a hologram of multiple images in simulations. Simulation results are useful to the evaluation of the performance by PSNR.

\section{A. MIE by $\mathrm{OSH}$}

In the first experiment, hologram data are measured by a physical OSH system as shown in Fig. 1. The hologram is derived from "star" and "heart" symbols positioned at $0.97 \mathrm{~m}$ and $1.17 \mathrm{~m}$ from the focal spot of lens $\mathrm{L}_{1}$, respectively. The two sections are located as shown in Fig. 7 (abridged sketch of the scanning part in the OSH system shown in Fig. 1). The scanner projects the combined beam of the laser onto the two sections. The beam passes through the two sections successively, and then the transmitted light is collected by a photodetector, which generates a scanned electrical signal. The electrical signal is then processed to obtain the holographic signal of the two scanned sections. Finally the holographic signal is stored in a computer as a complex hologram. The complex hologram is of the size $500 \times 500$ pixels with a pixel size of $20 \mu \mathrm{m} \times 20 \mu \mathrm{m}$. The complex hologram's real and imaginary parts are shown in Figs. 8(a) and 8(b), respectively. Then a sampling mask such as that shown in Fig. 3 is applied to the FT result of the hologram to obtain the compressed hologram data. Again, the sampling rate is at 0.25 , which means we take 1 out of 4 data points from the hologram, and sampling is nonuniform, as mentioned before.

Reconstruction of the compressed hologram is implemented according to Eq. (13). Reconstructed sections are shown in Fig. 9 , in which subfigures (a) and

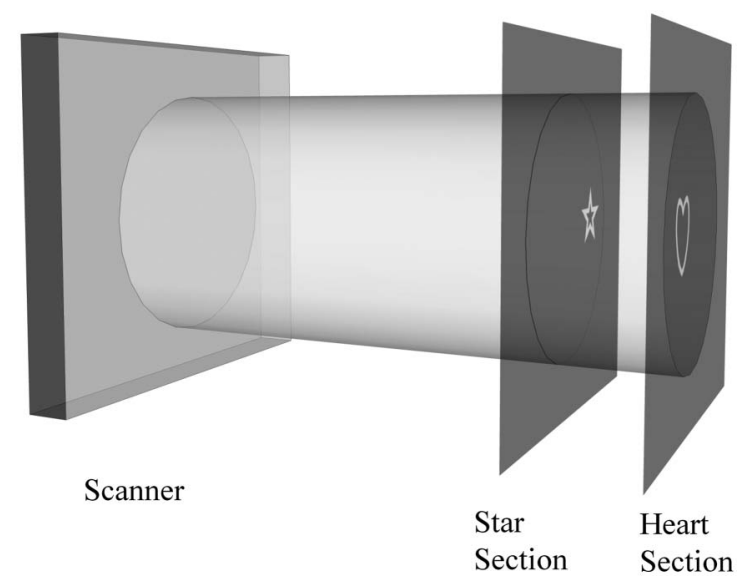

Fig. 7. Abridged sketch of the "star" and "heart" sections scanned in the OSH system. 


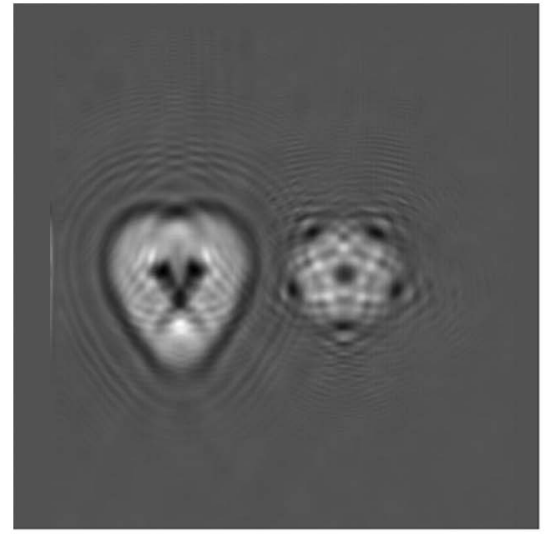

(a)

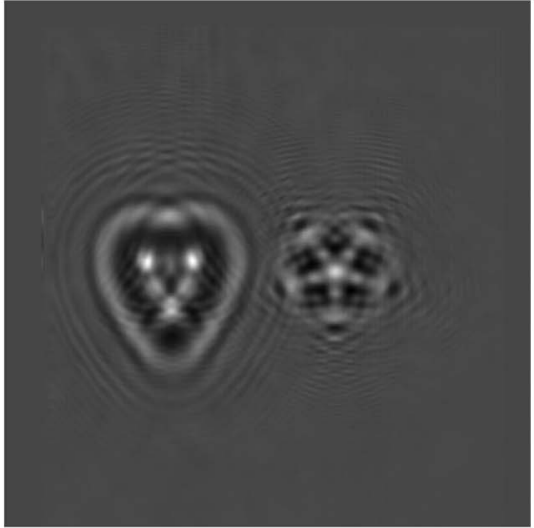

(b)

Fig. 8. (a) Real part and (b) imaginary part of the hologram.

(b) are sections recovered without compression using inverse imaging as in [17] directly. As the reconstruction shows, the "star" and "heart" are well recovered and there is little perceptible defocus noise left. The result verifies the reconstruction method, which works effectively to extract sections from the hologram while suppressing the defocus noise.

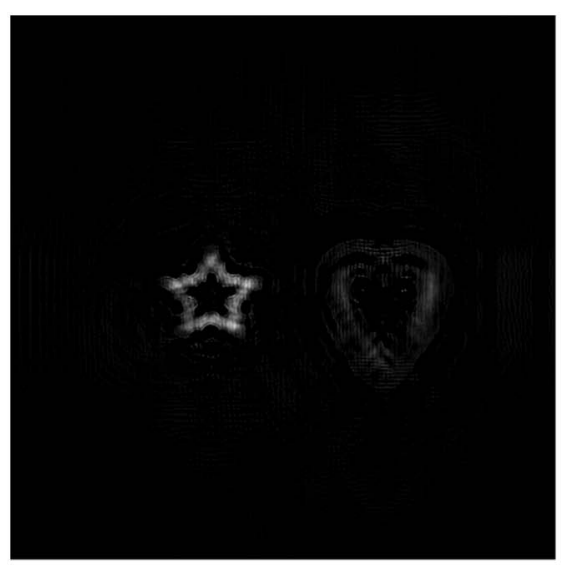

(a)

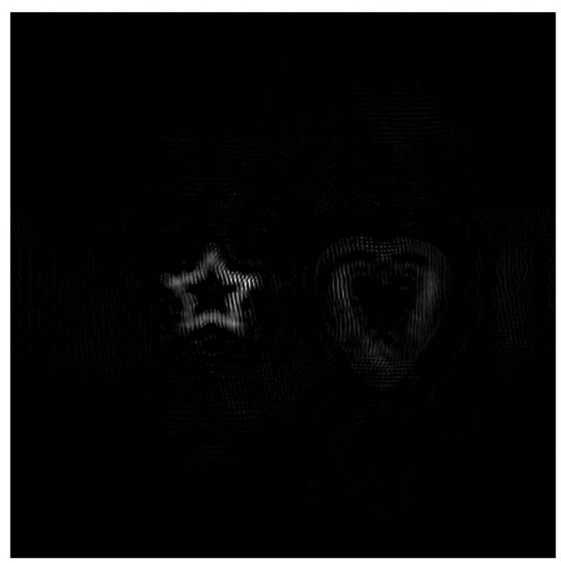

(c)
Reconstruction is also applied to a compressed hologram, in which the QCSR $=0.25$ and the quantization is int8. Figures $\underline{9(\mathrm{c})}$ and $\underline{9(\mathrm{~d})}$ show sections extracted from the hologram. Comparing the results in Figs. 9(a) and 9(b) with those in Figs. $\underline{\text { 9(c) }}$ and 9(d), we observe that the reconstruction of the compressed hologram also makes sections be recovered

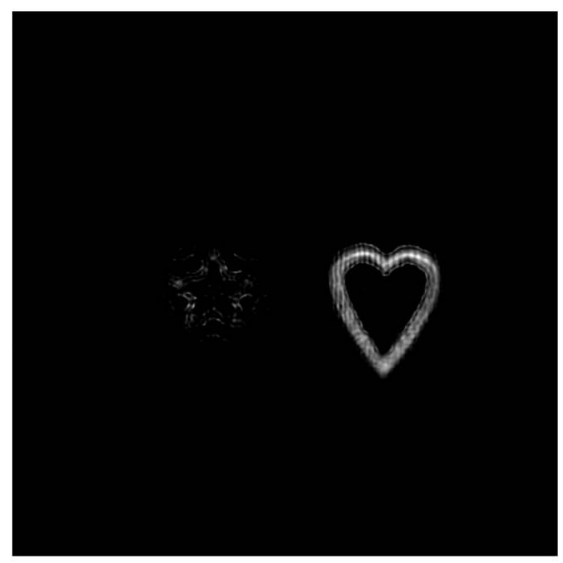

(b)

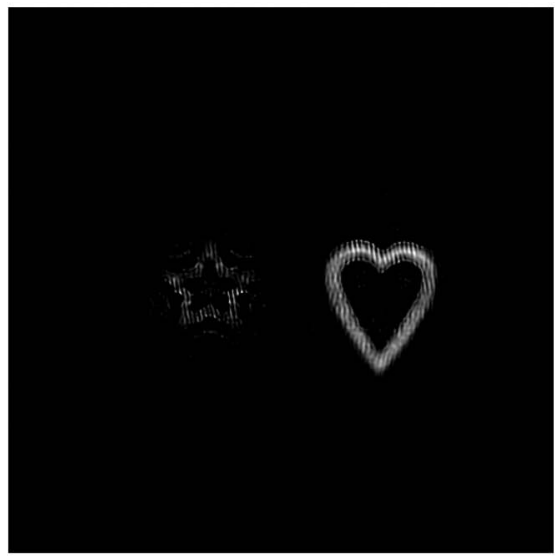

(d)

Fig. 9. (a), (b) Reconstructions of different sections are shown in by the inverse imaging method. (c), (d) Corresponding reconstructed sections with quantization $=$ int8, QCSR $=0.25$. 


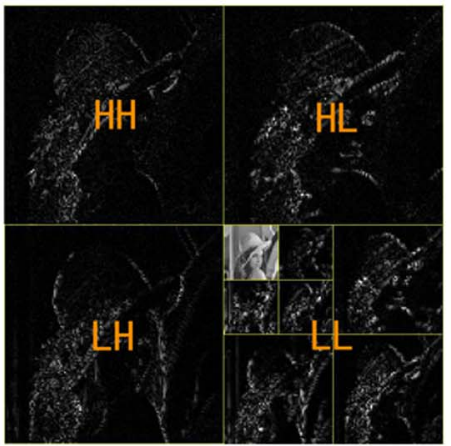

(a)

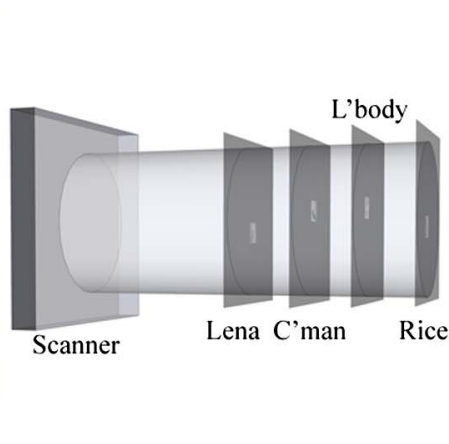

(b)

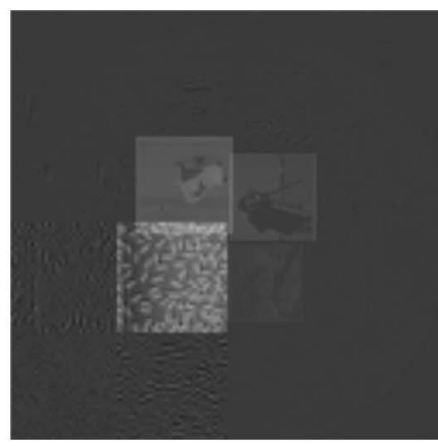

(c)

Fig. 10. (Color online) (a) DWT results showing LL and HH have been exchanged in location. (b) Scanning four level-3 decompositions by the OSH system. (c) The four subimages are collapsed onto a single plane to illustrate the nonoverlapping of the subimages.

clearly with defocus noise suppressed and gives the hologram data compressed quarterly.

\section{B. Holographic Encryption of Multiple Images}

In the previous optical experiment, it is worthwhile to note that the hidden images, "star" and "heart," are quite intuitive and are nonoverlapping. In what follows, we perform computer simulations using the principle of $\mathrm{OSH}$ for multiple natural images to test the feasibility of our proposed algorithm.

For overlapping images, it is necessary to involve preprocessing to handle the intensity distribution of each section of the image. Otherwise, sections adjacent to the scanner would block the beam from projecting on sections far away. We propose using a wavelet transform for preprocessing to decompose images and reduce overlapped information. Here the Daubechies D5 wavelet is chosen as it is good at picking up details in an image. After a three-level wavelet transform, low-frequency data with much information are arranged at the left upper corner (LL subimage) while high-frequency data are at the right lower corner (HH subimage) but with less information (see Fig. 5). Then we exchange the two quadrants, LL and $\overline{\mathrm{H}} \mathrm{H}$, with each other so that the coefficients at the low-frequency part at level 3 are scanned in the center, as shown in Fig. 10(a), to avoid the aliasing at boundaries in the scanning. Next, coefficients of the second, third, and fourth sections are rotated by $90^{\circ}, 180^{\circ}$, and $270^{\circ}$, respectively, to overcome overlapping. The effect of rotation is obvious in Fig. 10(c), in which low-frequency contents of each section occupy a quarter of the whole plane with less overlapping with others. The rotation reduces the overlapped information significantly. Finally, these organized coefficients are scanned by the OSH system as shown in Fig. 10(b). Figure 10(c) is the enlarged four decompositions collapsed onto a single plane to illustrate the nonoverlapping of the subimages. Note that although preprocessing is involved in the generation of a hologram, the critical part of the encryption is implemented by the $\mathrm{OSH}$ system and the preprocessing helps the beam to project onto all sections.

The hologram is shown in Fig. 11, where Fig. 11(a) is the real part and Fig. 11(b) is the imaginary part. The quantization of the hologram is set to int8 and the sampling rate is 50\%. According to Eq. (7), the $\mathrm{QCSR}=\frac{0.5 \times 1 \times 2}{4}=0.25$.

The reconstruction is carried out on the hologram. The third row of Fig. 12 shows reconstructed images from the compressed hologram. Compared with the original images shown in the first row of Fig. 12, the main information captured by these images is

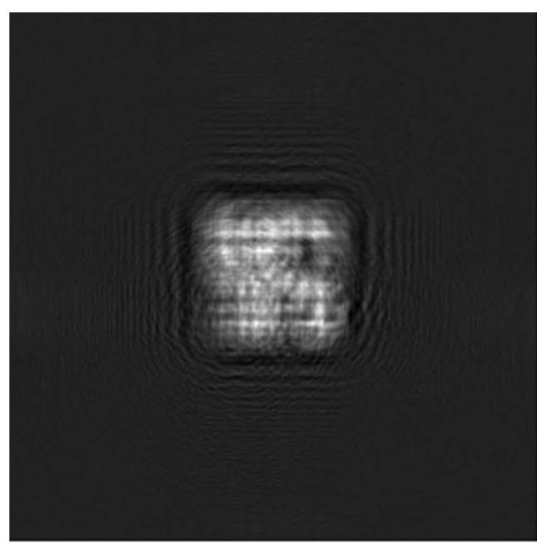

(a)

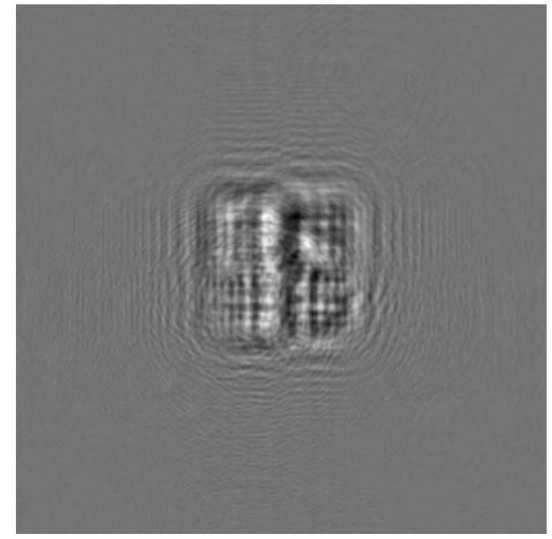

(b)

Fig. 11. Hologram of four images: (a) real part and (b) imaginary part. 


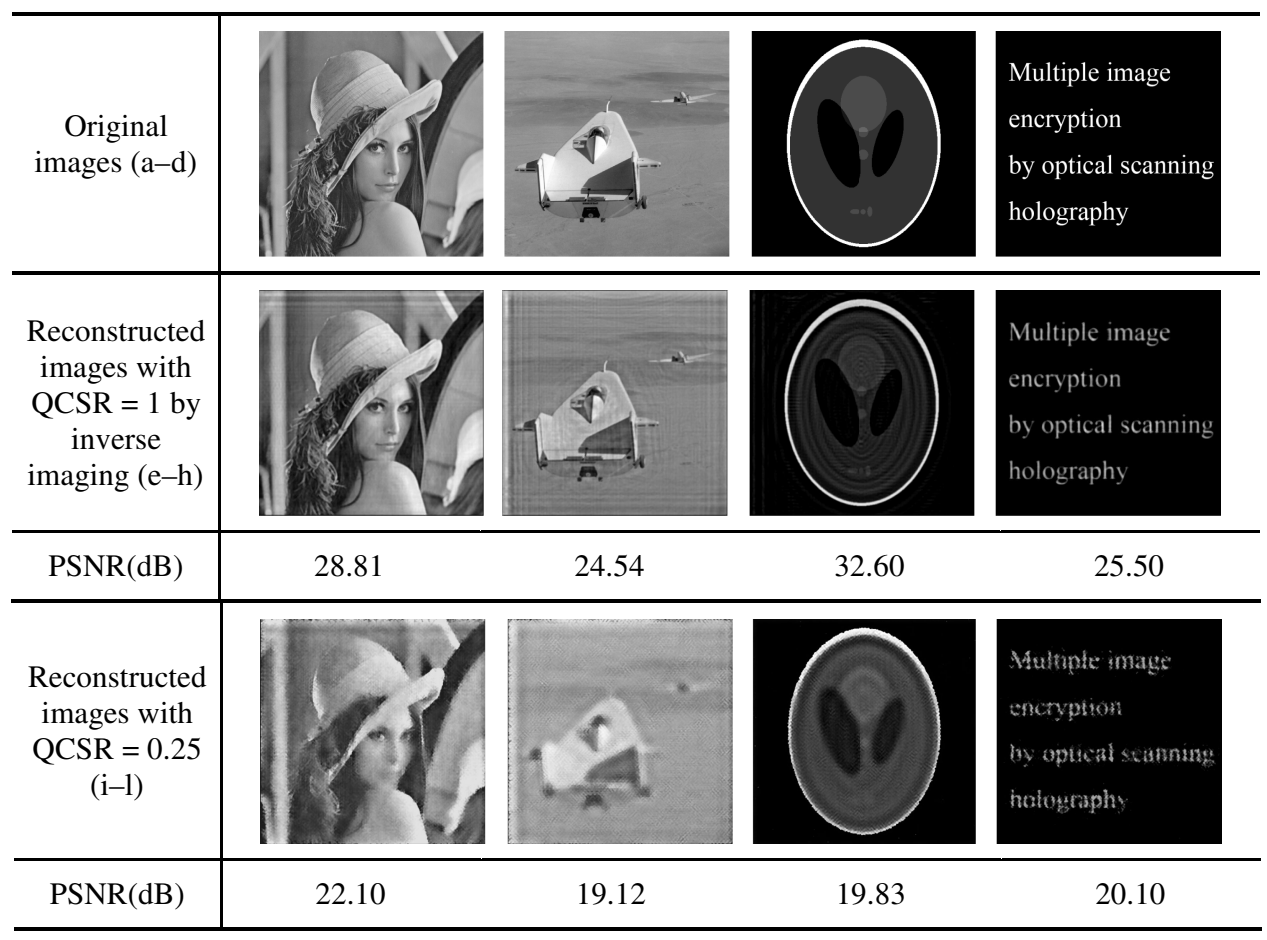

Fig. 12. Original and reconstructed images in the experiment.

reconstructed. The PSNR is close to or more than $20 \mathrm{dBm}$ in the reconstruction. Comparing these images with those in the second row of Fig. 12 from the noncompression hologram, PSNR is decreased but major edges are preserved for objects in these images. This demonstrates that the reconstruction works to decrypt multiple images from a compressed hologram by OSH.

\section{Conclusions}

We investigate both the compression and encryption of multiple images. MIE is achieved by the OSH system. The hologram with encrypted information is further explored with CS theory to achieve a compressed encryption, owing to the fact that the FT of the hologram corresponds to the 3D FT of the images at different places. The implementation of CS with the sparsity of natural images under a wavelet basis not only generates a compressed hologram but also ensures a decryption with desirable quality. The whole scheme of the encryption/ decryption of multiple images is verified by two experiments using real OSH data and simulated four-image hologram data. Their results are consistent and demonstrate that the reconstruction method works well to decrypt multiple images from a compressed hologram recorded by the OSH system.

The work is partly supported by the Chinese Academy of Sciences Visiting Professorship for Senior International Scientists (no. 2010T2G17). We also appreciate the support from the National Natural Science Foundation of China (nos. 81101082, 61121061), the National Basic Research Program of China (973 Program, no. 2007CB311203), and the 111 Project (no. B08004).

\section{References}

1. P. Refregier and B. Javidi, "Optical image encryption based on input plane and Fourier plane random encoding," Opt. Lett. 20, 767-769 (1995).

2. T.-C. Poon, T. Kim, and K. Doh, "Optical scanning cryptography for secure wireless transmission," Appl. Opt. 42 , 6496-6503 (2003).

3. G. Unnikrishnan, J. Joseph, and K. Singh, "Optical encryption by double-random phase encoding in the fractional Fourier domain," Opt. Lett. 25, 887-889 (2000).

4. Z. Liu, Y. Zhang, H. Zhao, M. A. Ahmad, and S. Liu, "Optical multi-image encryption based on frequency shift," Optik 122, 1010-1013 (2011).

5. G. Situ and J. Zhang, "Multiple-image encryption by wavelength multiplexing," Opt. Lett. 30, 1306-1308 (2005).

6. Z. Liu and S. Liu, "Double image encryption based on iterative fractional Fourier transform," Opt. Commun. 275, 324-329 (2007).

7. X. F. Meng, L. Z. Cai, M. Z. He, G. Y. Dong, and X. X. Shen, "Cross-talk free image encryption and watermarking by digital holography and random composition," Opt. Commun. 269, 47-52 (2007).

8. S. L. Diab, "Developing an algorithm for compression, multiplexing and enhancement of multiple images," Opt. Laser Technol. 43, 838-847 (2011).

9. O. Matoba, T. Nomura, E. Perez-Cabre, M. S. Millan, and B. Javidi, "Optical techniques for information security," Proc. IEEE 97, 1128-1148 (2009).

10. Y. Frauel, T. J. Naughton, O. Matoba, E. Tajahuerce, and B. Javidi, "Three-dimensional imaging and processing using computational holographic imaging," Proc. IEEE 94, 636-653 (2006).

11. S. Soontaranon and J. Widjaja, "Holographic image encryption by using random phase modulation of plane wave," Opt. Lasers Eng. 48, 994-999 (2010).

12. J. F. Heanue, M. C. Bashaw, and L. Hesselink, "Encrypted holographic data storage based on orthogonal-phase-code multiplexing," Appl. Opt. 34, 6012-6015 (1995). 
13. P. Tsang, W. K. Cheung, T. Kim, Y. S. Kim, and T.-C. Poon, "Low-complexity compression of holograms based on delta modulation," Opt. Commun. 284, 2113-2117 (2011).

14. W.-C. Su, C.-C. Sun, Y.-C. Chen, and Y. Ouyang, "Duplication of phase key for random-phase-encrypted volume holograms," Appl. Opt. 43, 1728-1733 (2004).

15. C.-C. Sun and W.-C. Su, "Three-dimensional shifting selectivity of random phase encoding in volume holograms," Appl. Opt. 40, 1253-1260 (2001).

16. C. Denz, K. O. Mueller, F. Visinka, and T. T. Tschudi, "Digital volume holographic data storage using phase-coded multiplexing," Proc. SPIE, 142-147 (1999).

17. X. Zhang, E. Y. Lam, and T.-C. Poon, "Reconstruction of sectional images in holography using inverse imaging," Opt. Express 16, 17215-17226 (2008).

18. E. Y. Lam, X. Zhang, H. Vo, T.-C. Poon, and G. Indebetouw, "Three-dimensional microscopy and sectional image reconstruction using optical scanning holography," Appl. Opt. 48, H113-H119 (2009).

19. X. Zhang, E. Y. Lam, T. Kim, Y. S. Kim, and T.-C. Poon, "Blind sectional image reconstruction for optical scanning holography," Opt. Lett. 34, 3098-3100 (2009).
20. J. Ke, T.-C. Poon, and E. Y. Lam, "Depth resolution enhancement in optical scanning holography with a dual-wavelength laser source," Appl. Opt. 50, H285-H296 (2011).

21. T.-C. Poon, "Scanning holography and two-dimensional image processing by acousto-optic two-pupil synthesis," J. Opt. Soc. Am. A 2, 521-527 (1985).

22. T.-C. Poon and G. Indebetouw, "Three-dimensional point spread functions of an optical heterodyne scanning image processor," Appl. Opt. 42, 1485-1492 (2003).

23. T. C. Poon, Optical Scanning Holography with MATLAB (Springer-Verlag, 2007).

24. J. W. Goodman, Introduction to Fourier Optics, 3rd ed. (Roberts \& Co., 2005).

25. X. Zhang and E. Y. Lam, "Edge-preserving sectional image reconstruction in optical scanning holography," J. Opt. Soc. Am. A 27, 1630-1637 (2010).

26. Y. Rivenson, A. Stern, and B. Javidi, "Compressive Fresnel holography,” J. Display Technol. 6, 506-509 (2010).

27. L. He and L. Carin, "Exploiting structure in wavelet-based Bayesian compressive sensing," IEEE Trans. Signal Process. 57, 3488-3497 (2009).

28. E. Candès and J. Romberg, "Sparsity and incoherence in compressive sampling," Inverse Probl. 23, 969-985 (2007). 